\title{
High field MRI in the diagnosis of multiple sclerosis: high field-high yield?
}

\author{
Mike P. Wattjes • Frederik Barkhof
}

Received: 30 January 2009 / Accepted: 11 February 2009 / Published online: 11 March 2009

(C) The Author(s) 2009. This article is published with open access at Springerlink.com

\begin{abstract}
Following the approval of the U.S. Food and Drug Administration (FDA), high field magnetic resonance imaging (MRI) has been increasingly incorporated into the clinical setting. Especially in the field of neuroimaging, the number of high field MRI applications has been increased dramatically. Taking advantage on increased signal-to-noise ratio (SNR) and chemical shift, higher magnetic field strengths offer new perspectives particularly in brain imaging and also challenges in terms of several technical and physical consequences. Over the past few years, many applications of high field MRI in patients with suspected and definite multiple sclerosis (MS) have been reported including conventional and quantitative MRI methods. Conventional pulse sequences at $3 \mathrm{~T}$ offers higher lesion detection rates when compared to $1.5 \mathrm{~T}$, particularly in anatomic regions which are important for the diagnosis of patients with MS. MR spectroscopy at $3 \mathrm{~T}$ is characterized by an improved spectral resolution due to increased chemical shift allowing a better quantification of metabolites. It detects significant axonal damage already in patients presenting with clinically isolated syndromes and can quantify metabolites of special interest such as glutamate which is technically difficult to quantify at lower field strengths. Furthermore, the higher susceptibility and SNR offer advantages in the field of functional MRI and diffusion tensor imaging. The recently introduced new generation of ultra-high field systems beyond $3 \mathrm{~T}$ allows scanning in submillimeter resolution and gives new insights into in vivo MS pathology on MRI. The objectives of this
\end{abstract}

M. P. Wattjes $(\bowtie) \cdot$ F. Barkhof

MS Center Amsterdam, Department of Radiology,

VU University Medical Center,

De Boelelaan 1117,

1081 HV Amsterdam, The Netherlands

e-mail: m.wattjes@vumc.nl article are to review the current knowledge and level of evidence concerning the application of high field MRI in MS and to give some ideas of research perspectives in the future.

Keywords Multiple sclerosis · Clinically isolated syndrome $\cdot$ High field magnetic resonance imaging . MR spectroscopy · Diffusion tensor imaging .

Functional magnetic resonance imaging

\section{Introduction}

Multiple sclerosis (MS) is the most common chronic inflammatory disease of the central nervous system (CNS) in young adults with a prevalence of 1:1,000 people in Europe [1]. Pathologically, MS is a heterogenic chronic immune-mediated inflammatory disorder of the CNS disseminated in space and in time which is characterized by focal and diffuse areas of demyelination within the white and gray matter associated with axonal damage and loss [2]. Magnetic resonance imaging (MRI) is the most sensitive diagnostic tool in the detection of focal and diffuse changes of MS in vivo and has become the most important paraclinical method in the diagnosis of MS within diagnostic criteria [3-5]. The so-called McDonald criteria allow a diagnosis of MS based on MRI findings as early as 3 months after the first clinical event without further clinical relapses [6, 7]. Furthermore, in patients presenting with a clinically isolated syndrome (CIS) suggestive of MS, MRI has gained an important prognostic value in terms of predicting the conversion to clinically definite MS and long-term disability [8-10].

Currently, most of the MRI protocols for clinical and research purposes are based on magnetic field strengths up 
to $1.5 \mathrm{~T}$. Since the US Food and Drug Administration approved magnetic field strength up to $8 \mathrm{~T}$ as having nonsignificant risk [11], high field MRI systems are increasingly incorporated into the clinical setting especially in the field of neuroimaging leading to new possibilities and also challenges in many directions.

The aims of this review are to provide a comprehensive overview regarding the current level of knowledge and evidence in terms of high field MRI in the diagnosis and disease monitoring of MS and to discuss new directions of high field MR imaging in MS.

\section{High field MRI: technical considerations and its implications for clinical applications}

Moving to higher magnetic field strengths implies many technical and physical changes including the signal-tonoise ratio (SNR), tissue relaxation times, susceptibility, Larmor frequency, chemical shift, $\mathrm{B}_{1}$ heterogeneity, and radiofrequency power deposition. All the factors have major consequences for the adaptation of imaging protocols from lower to higher magnetic field strengths and the implementation of new protocols in many different ways $[12,13]$.

The SNR is considered to be approximately linearly related to the magnetic field strength $\left(S / N \sim B_{0}\right)$. In other words, a doubling of the magnetic field from 1.5 to $3 \mathrm{~T}$ should double the signal. The increase in SNR is one of the major advantages of MRI at higher field which can be invested either in reducing scan time (e.g., by applying parallel imaging techniques and/or variable flip angles, longer echo trains) or in an increase of spatial resolution (smaller voxel size) [14, 15].

Tissue relaxation times are substantially depending on the static magnetic field $\left(B_{0}\right)$. The T1 (longitudinal) relaxation time is significantly increasing when moving to higher field strengths, increasing by approximately $40 \%$ in the white matter at $3 \mathrm{~T}$ when compared to $1.5 \mathrm{~T}$. This has major consequences for gadolinium (Gd)-enhanced T1weighted sequences because this $\mathrm{T} 1$ prolongation at higher fields pronounces the T1 shortening effect of Gd-based contrast media leading to greater post-contrast signal of the enhancing tissues [16]. Therefore, this higher T1 effect might allow a lower dosage of contrast media at $3.0 \mathrm{~T}$. The change of the T2 relaxation time is less pronounced and ranging up to $5 \%[12,17]$.

On the other hand, $\mathrm{T} 2 *$ relaxation time and therefore $\mathrm{T} 2 *$ based sequences are remarkably influenced by higher $B_{0}$ because of the substantial increase of susceptibility $(\Delta B \sim$ $B_{0}$ ). Besides its negative consequences in terms of image quality, the increase in susceptibility at higher fields can be used for a better application of susceptibility-weighted (perfusion) MR imaging and blood oxygen level-dependent functional MRI (BOLD).

Chemical shift also increases proportionally with the applied $B_{0}\left(\mathrm{CS} \sim B_{0}\right)$. This offers great potentials for MR spectroscopy due to an increased spectral resolution. The increase of chemical shift in combination with higher SNR offers the possibility for a better quantification of metabolite concentrations especially in terms of sensitivity and reproducibility [18, 19].

Major drawbacks of imaging at higher magnetic fields are $B_{1}$ heterogeneity and radiofrequency (RF) power deposition. Especially the RF power deposition in terms of the specific absorption rate (SAR) is of special interest concerning safety aspects. SAR increases with the square of $B_{0}\left(\mathrm{SAR} \sim B_{0}{ }^{2}\right)$. There are strict regulations of RF heating in the tissue available and the SAR may not exceed $4 \mathrm{~W} / \mathrm{kg}$ within $15 \mathrm{~min}$ [20]. This limit is more likely to be reached at higher magnetic fields especially by using pulse sequences with a high SAR potential such as turbo(fast)spin echo (TSE) sequences. The implementation of parallel imaging techniques and variable flip angles can be helpful to address this issue [14].

\section{Conventional high field MRI at $3 \mathrm{~T}$ in multiple sclerosis}

Magnetic field strength-dependent lesion load measurement

The influence of the magnetic field strength on the detection rate has already been of great interest since the introduction of 1.5-T MRI scanners in the early 1990s. Table 1 gives an overview of studies investigating the possible influence of higher magnetic field strengths on the detection of focal inflammatory lesions on CIS and MS patients.

The first studies comparing magnetic field strengths of 0.5 and $1.5 \mathrm{~T}$ can be considered as inconclusive [21-23]. Two studies showed significantly higher lesion load measurements at $1.5 \mathrm{~T}$ when compared to lower field strengths especially in the infratentorial region [21, 22], however, without any implications on this diagnosis of MS according to the Poser criteria [24]. These results could not be confirmed by the following study including a larger cohort of 132 patients with suspected or definite MS [23].

Since the new generation of clinical high field scanners mainly operating at $3 \mathrm{~T}$ has been incorporated into the clinical setting, the question of possible field strengthdependent different lesion load measurements in suspected or definite MS revived again. The first evidence that the new high field MRI generation provides higher lesion load measurements in MS patients was brought up by an intraindividual comparative study between 1.5 and $4 \mathrm{~T}$. Keiper et al. showed a $45 \%$ increase of $\mathrm{T} 2$ lesions using a 
Table 1 Overview of published studies investigating the possible influence of increasing magnetic field strengths on the detection of acute and chronic inflammatory lesions in patients with CIS or definite MS.

\begin{tabular}{|c|c|c|c|}
\hline Author, reference & Field strengths tested $(\mathrm{T})$ & Patients; number & Results \\
\hline Schima et al. [21] & $0.5,1.5$ & CIS, definite MS; $n=27$ & $9.1 \% \uparrow$ of $\mathrm{T} 2$ lesions \\
\hline Lee et al. [22] & $0.5,1.5$ & Definite, suspected MS; $n=132$ & No significant increase of lesion load \\
\hline Filippi et al. [23] & $0.5,1.0,1.5$ & Definite MS; $n=20$ & Significant increase of $\mathrm{T} 2$ lesion volume \\
\hline Keiper et al. [25] & $1.5,4.0$ & Definite MS; $n=15$ & $45 \% \uparrow$ of $\mathrm{T} 2$ lesions \\
\hline Sicotte et al. [26] & $1.5,3.0$ & Definite MS; $n=25$ & $\begin{array}{l}21 \% \uparrow \text { of } \mathrm{T} 1 \mathrm{Gd}+\text { lesions, } 54 \%, 10.7 \% \uparrow \text { of } \mathrm{T} 1 \mathrm{Gd}+ \\
\mathrm{PD} \text { lesion volume }\end{array}$ \\
\hline Wattjes et al. [27] & $1.5,3.0$ & CIS; $n=40$ & $7.5 \% \uparrow$ of $\mathrm{T} 1 \mathrm{Gd}+$ lesions $13 \% \uparrow$ lesions, $\mathrm{T} 2$ and FLAIR, \\
\hline Nielsen et al. [28] & $1.5,3.0$ & Definite MS, CIS; $n=28$ & $\begin{array}{l}24 \% \uparrow \text { of } \mathrm{T} 1 \mathrm{Gd}+\text { lesions, } 26.5 \% \text { of FLAIR lesions per } \\
\text { patient }\end{array}$ \\
\hline Bachmann et al. [29] & $1.5,3.0$ & Definite MS; $n=22$ & 41.6.\% $\uparrow$ lesions on FLAIR \\
\hline
\end{tabular}

$M S$ multiple sclerosis, $C I S$ clinically isolated syndromes suggestive of MS, $T$ Tesla, $G d+$ gadolinium-enhancing, FLAIR fluid-attenuated inversion recovery, $\uparrow$ increase

4-T compared to a 1.5-T scanner [25]. This higher sensitivity in the detection of focal MS lesions and/or lesion volumes could be conclusively confirmed by the subsequent studies comparing field strengths between 1.5 and $3 \mathrm{~T}$ including a total number 115 patients scanned with MRI scanners of three different vendors [26-29]. The increase in lesion numbers on T2/fluid-attenuated inversion recovery (FLAIR) images ranged from $13 \%$ to $41.6 \%$. Concerning Gd-enhancing lesions the increase in sensitivity amounts to $7.5-24 \%$. The largest comparative study of 1.5 and $3 \mathrm{~T}$ including 40 patients presenting with a CIS presented a subgroup analysis of different anatomic locations. The authors found a pronounced increase of $\mathrm{T} 2$ and FLAIR lesion numbers in anatomic regions which are important for the diagnosis of MS within diagnostic criteria and prognostic purposes in terms of prediction of long-term disability, namely in the juxtacortical, periventricular, and of course in the infratentorial region (Fig. 1) [27]. Further analysis revealed that based on a multisequence protocol including a Gd-enhanced T1-weighted sequence, $27.5 \%$ of those patients with more lesions on the $3 \mathrm{~T}$ fulfilled one additional MRI criterion [30]. Acknowledging that these MRI criteria have a strong predictive value concerning the conversion to definite MS, patients are likely to receive a different (worse) prognostic classification based on a 3-T examination when compared to $1.5 \mathrm{~T}[8,9]$. However, including neurological examinations (cerebrospinal fluid (CSF) examinations, etc.) into the analysis, it became clear that these higher lesion load measurements on $3 \mathrm{~T}$ do not necessarily lead to different classifications according to the clinical International Panel (IP) criteria for MS. Only one single patient in this study received the diagnosis of dissemination in space based on the $3 \mathrm{~T}$ but not on the 1.5-T examination [30]. Additionally, a short-term followup study of this patient cohort clearly demonstrated that higher lesion load measurements on $3 \mathrm{~T}$ versus $1.5 \mathrm{~T}$ do not lead to an earlier diagnosis of MS [31], neither according to the revised IP criteria nor to the recently introduced Swanton criteria [7, 32].

In contrast to the high amount of data in terms of high field MRI applications in the brain, almost no experience exists regarding spinal cord imaging in MS using MRI field strengths beyond $1.5 \mathrm{~T}$. A recently published study is the first demonstrating the applicability of 3-T MRI in MS when compared to $1.5 \mathrm{~T}$. The authors investigated 32 patients using a non-cardiac gated, single late echo T2weighted sequence at both field strengths. Although the primary aim of this study was not a side-by-side comparison of $3 \mathrm{~T}$ with $1.5 \mathrm{~T}$ in terms of lesion detectability, this study showed no significant higher focal lesion load measurements in the spinal cord on $3 \mathrm{~T}$. Furthermore, the $\mathrm{T} 2$ lesion load on $3 \mathrm{~T}$ did not correlate better to clinical outcome measures such as disability when compared to T2 lesion load measurements obtained at $1.5 \mathrm{~T}$ [33].

\section{Pulse sequences}

According to recent published guidelines, the conventional MRI protocol for patients with suspected or definite MS is performed as a multisequence imaging protocol, including axial proton density (PD)/T2-weighted, sagittal FLAIR, and axial Gd-enhanced T1-weighted sequences [34]. These guidelines are based on experiences made on magnetic field strengths up to $1.5 \mathrm{~T}$. At lower $(0.5-1.0 \mathrm{~T})$ and standard field strengths $(1.5 \mathrm{~T})$, it became obvious that the sensitivity of T2-weighted pulse sequences in the detection of focal MS lesions is dependent on the anatomic region. T2-weighted (turbo) spin echo (SE) sequences are characterized by a high sensitivity in the detection of infratentorial lesions, whereas the sensitivity is rather limited in the supratentorial brain, 


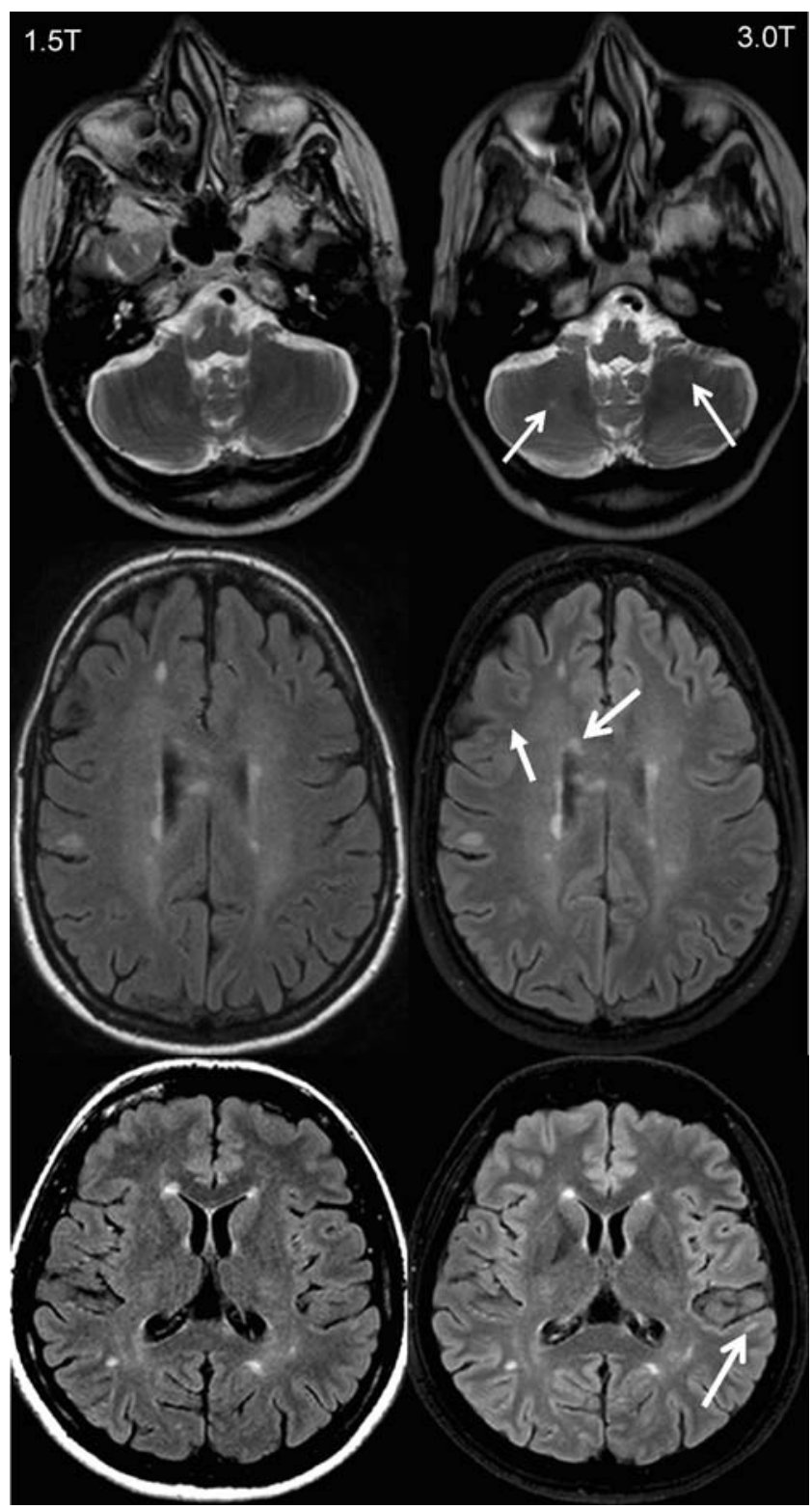

Fig. 1 Axial T2-weighted TSE (upper row) and FLAIR (middle and bottom rows) sequences from patients with relapsing-remitting MS. Compared to $1.5 \mathrm{~T}$, the images based on 3-T examinations (right column) show a higher conspicuity and identifiability of lesions (arrows) in several anatomic regions of the brain especially the infratentorial (upper row), periventricular (middle row), and juxtacortical (bottom row) white matter. Please note also that the higher SNR of $3 \mathrm{~T}$ leads to a better visualization of small lesions (middle row, closed arrow) which are likely to disappear in the noise of the white matter at lower magnetic field strengths

particularly in the juxtacortical and periventricular white matter. FLAIR sequences provide higher lesion detection rates in the juxtacortical and periventricular white matter but lack sensitivity in the detection of lesions in the posterior fossa and thalamus [35-39].

Moving to $3 \mathrm{~T}$ imaging, evidence is emerging that this phenomenon of region-dependent lesion detectability is somehow disappearing. An intraindividual comparative study of T2-weighted TSE and FLAIR sequences at $3 \mathrm{~T}$ including 49 patients presenting with CIS or definite MS showed no significant changes concerning the sensitivity in the detection of infratentorial lesions (Fig. 2) [40]. However, concerning the supratentorial brain, FLAIR remains superior to the T2 TSE, especially regarding the periventricular and juxtacortical regions. The underlying reason of this finding is not well understood, since contrast measurements revealed no significant differences between both sequences in the posterior fossa. Perhaps the FLAIR sequence, characterized by low SNR levels infratentorially at $1.5 \mathrm{~T}$, benefits most from the higher SNR provided by $3 \mathrm{~T}$.

Given the fact that the FLAIR sequence at $3 \mathrm{~T}$ has the highest overall sensitivity in the detection of inflammatory lesions without relevant disadvantages regarding the posterior fossa, the crucial question rises up whether a FLAIR sequence at $3 \mathrm{~T}$ alone might be sufficient within a MS imaging protocol. From our perspective, these questions cannot be answered sufficiently yet because the current data are only based on one study using a MRI device of one vendor. Additional studies including MRI systems of other vendors are necessary to validate these initial findings.

A new group of pulse sequences, which has been recently introduced into the field of MS research, is double inversion recovery (DIR) sequences. These sequences are characterized by two inversion pulses achieving a simultaneous suppression of two different tissue types. Dependent on the chosen inversion time, it is possible to simultaneously suppress the white matter and CSF ("gray matter only") or the gray matter and CSF ("white matter only"; Fig. 3) [41]. The first approaches using a 2D DIR application for brain imaging were quite promising regarding many disease entities including inflammatory, vascular, and neoplastic diseases. However, DIR at $1.5 \mathrm{~T}$ was never established into the clinical routine probably due to the relatively low SNR due to the application of two inversion pulses, higher propensity of artefacts and longer scan time [42, 43]. Recently, a 3D application at $1.5 \mathrm{~T}$ has been introduced and evaluated concerning the detection of focal gray matter lesions in MS patients [44]. This study shows that 3D DIR is able to detect significantly more intracortical lesions in MS, a lesion type seen abundantly in MS patients but difficult to detect with conventional pulse sequences such as PD/T2 and FLAIR [45]. Currently, DIR applications are used to detect and quantify cortical lesion load in MS patients as well as correlate these measures with subtypes, outcome measures, and clinical presentations of MS patients [46, 47]. DIR applications at higher magnetic field strengths may overcome major problems of DIR applications at standard field strengths $(1.5 \mathrm{~T})$ such as low SNR values and therefore long acquisition times. So far, 
Fig. 2 Axial FLAIR (top row) and T2-weighted TSE (bottom row) from a patient with relapsing-remitting MS obtained at $1.5 \mathrm{~T}$ (left column) and $3 \mathrm{~T}$ (right column) showing multiple focal abnormalities in the cerebellum (arrows). At $1.5 \mathrm{~T}$, we can observe a higher conspicuity of the lesion on the T2 TSE sequences compared to the corresponding FLAIR image. Moving to $3 \mathrm{~T}$, the conspicuity and detection rate of focal MS lesions is equal on both sequences. Please note also the higher sensitivity using $3 \mathrm{~T}$ instead of $1.5 \mathrm{~T}$, in particular on the FLAIR images due to higher SNR

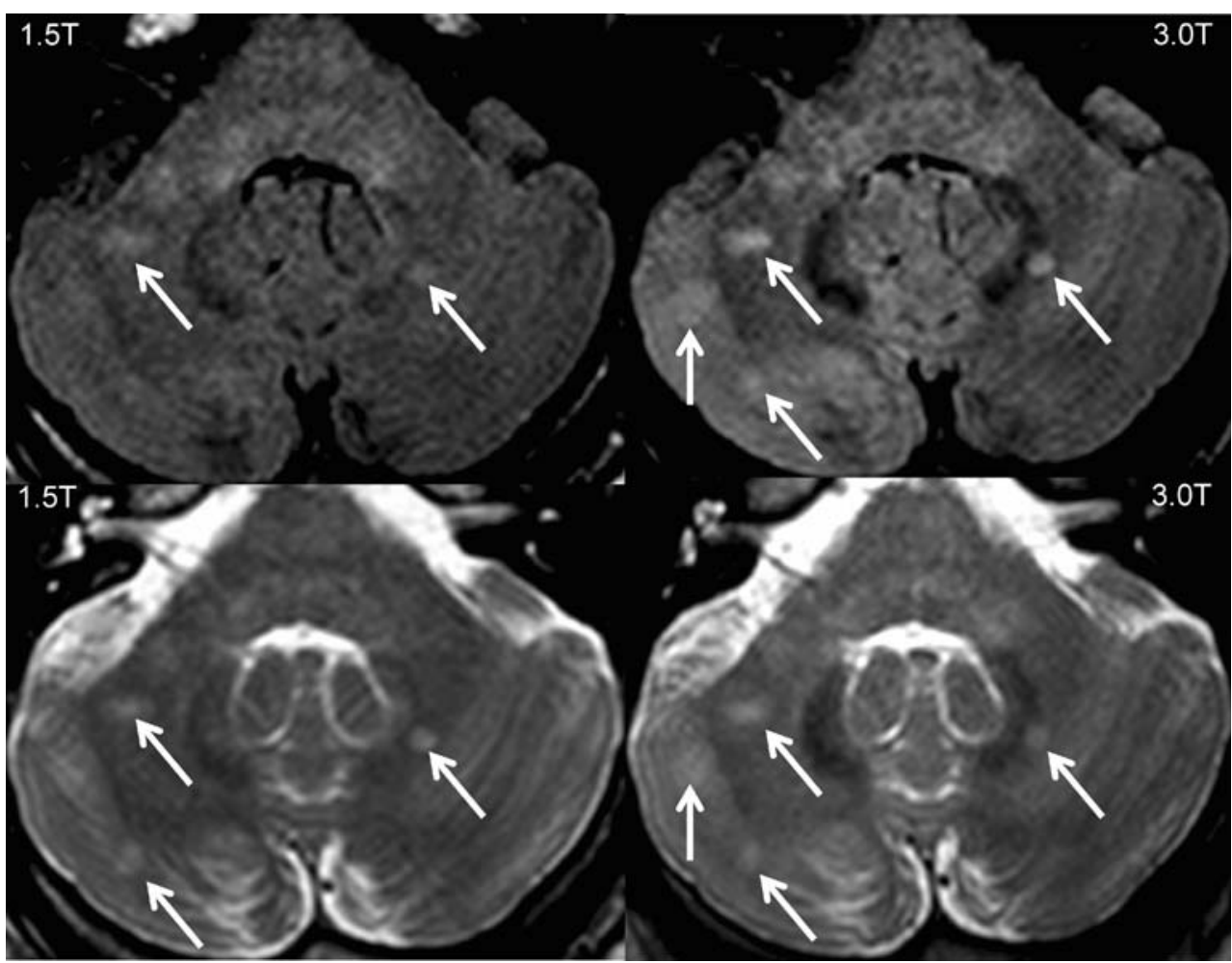

there is only one DIR study at $3 \mathrm{~T}$ available investigating the diagnostic value in the detection of MS lesions [48]. DIR at $3 \mathrm{~T}$ seems to be the sequence with the highest overall sensitivity in the detection of focal MS lesions when compared to T2 TSE and FLAIR. DIR at $3 \mathrm{~T}$ detected significantly more lesions than T2 TSE and FLAIR. Regarding specific anatomic locations, DIR showed comparable results for the juxtacortical, mixed white mattergray matter and periventricular lesions in comparison to FLAIR but significantly higher detection rates in the infratentorial regions when compared to both T2 TSE and FLAIR (Fig. 4). This finding makes this sequence particularly valuable for CIS patients since we know that infratentorial lesions have a certain prognostic relevance in terms of prediction of long-term disability [10]. There are two good explanations for this higher sensitivity of $3 \mathrm{~T}$ DIR concerning infratentorial lesions. First of all the contrast ratio between lesions and normal-appearing white matter (NAWM) was significant better for the DIR when compared to the T2 TSE and FLAIR. Secondly, similar to the experiences with FLAIR at $3 \mathrm{~T}$, DIR might better take advantage from the higher signal provided by $3 \mathrm{~T}$ especially in the posterior fossa. Due to the better contrast of gray matter versus white matter, many lesions classified as juxtacortical on the FLAIR and T2 TSE sequences were (re)classified as cortical or at least mixed gray matter-white matter lesions which resulted in a sort of "reclassification phenomenon" similar to experiences at $1.5 \mathrm{~T}$ (Fig. 5) [44, 48].

Fig. 3 Axial sections through the supratentorial brain of a healthy volunteer using a "double inversion recovery" sequence at $3 \mathrm{~T}$ (measured voxel size $3 \times 1 \times 1 \mathrm{~mm}$ ). The cerebrospinal fluid as well as the white matter is simultaneously attenuated leading to a high contrast and delineation between the white matter and gray matter

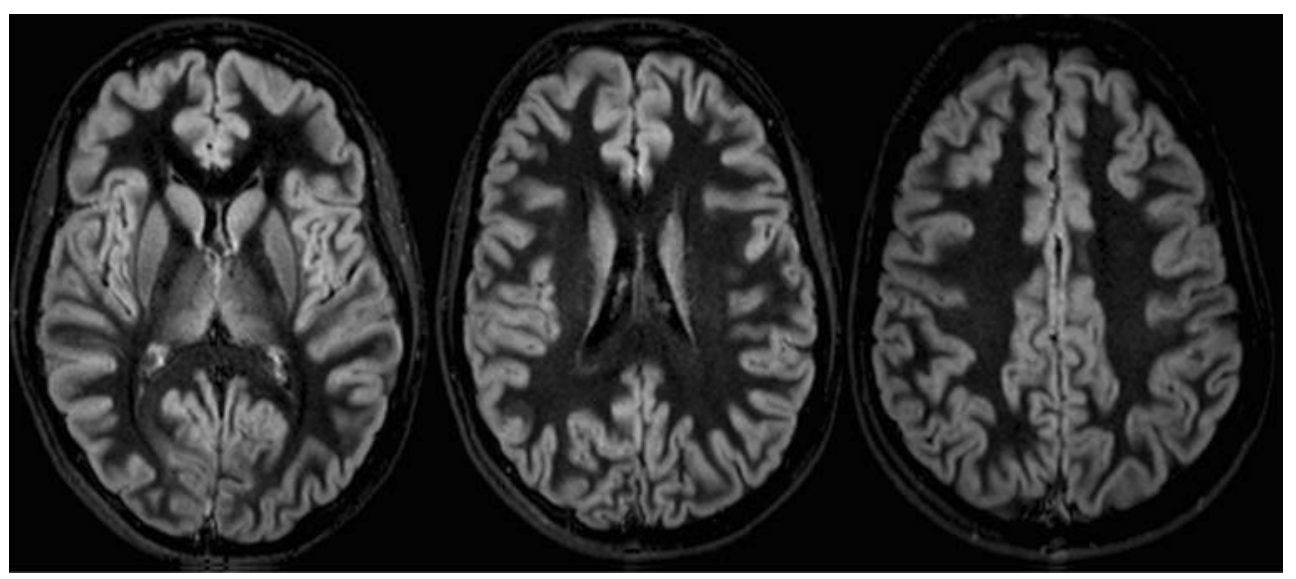


Fig. 4 Axial sections of $3 \mathrm{~T} 2 \mathrm{D}$ FLAIR (left column), T2-weighted TSE (middle column), and DIR (right column) sequences of patients with relapsing-remitting MS. Using DIR, more infratentorial lesions can be detected when compared to FLAIR and T2 TSE. Upper row: A focal lesion in the right mesencephalon touching the periaqueductal gray matter (arrow) could be easily detected on the DIR but was not prospectively identified on the corresponding FLAIR and T2 TSE images. Bottom row: A focal lesion in the right cerebellum is clearly visible on the DIR sequence with high signal intensity (arrow) but not visible on the corresponding FLAIR and T2 TSE images

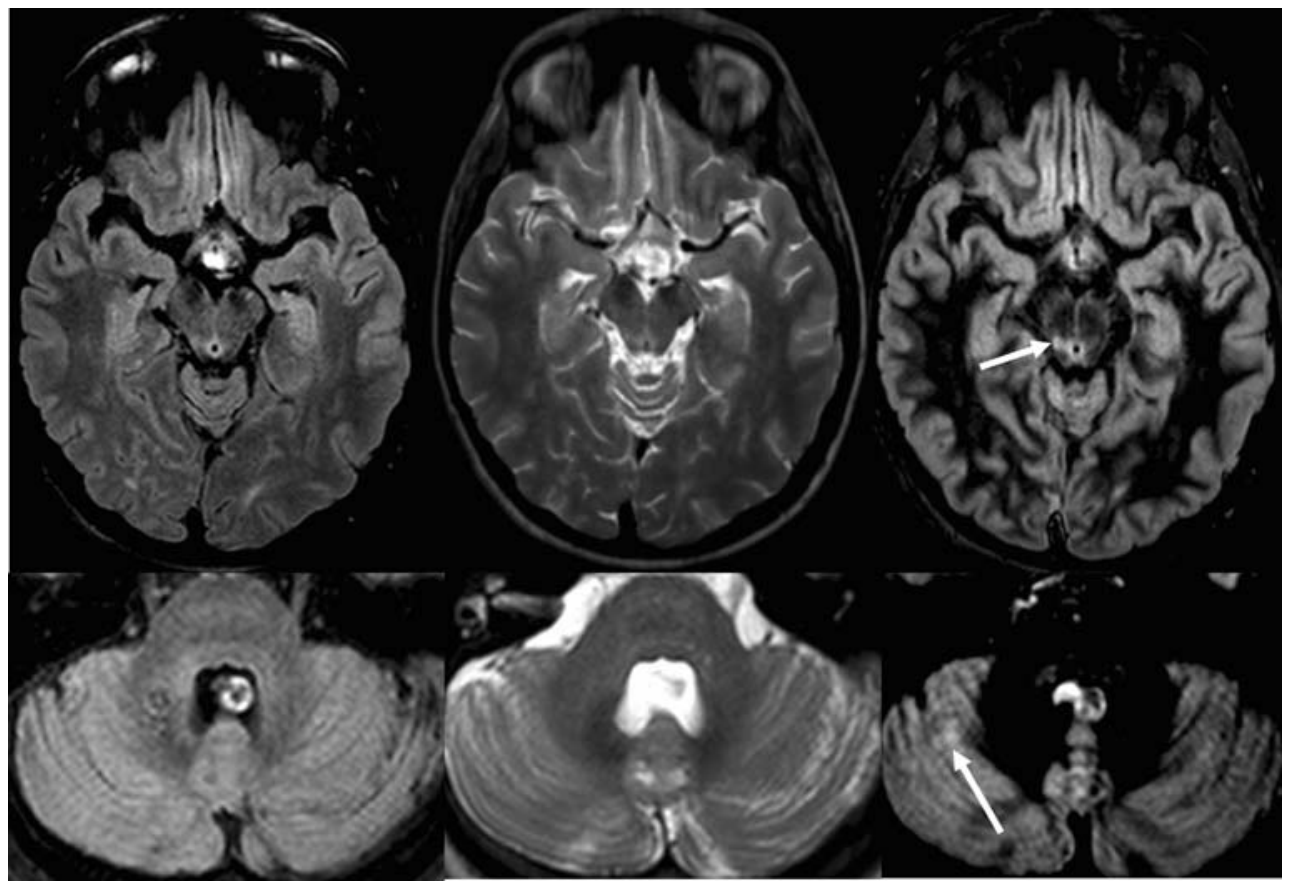

In addition, the higher SNR at $3 \mathrm{~T}$ can be invested into "speed-optimized" pulse sequences without losing diagnostic quality and sensitivity in the detection of MS lesions (Fig. 6). A study using a combination of T2-weighted fast spin echo, variable flip angle refocusing and half-Fourier acquisition, and parallel imaging achieved a reduction of the scan time from 2:02 $\mathrm{min}$ to $11 \mathrm{~s}$ (axial plane) and 3:24 min to $29 \mathrm{~s}$ (sagittal plane), respectively, without a relevant impairment in sensitivity. Still $88 \%$ of the 208 lesions detected on the standard T2 TSE sequence remained visible on fast T2 sequence [49].
3D applications are increasingly being used at standard field strengths with the use of signal saving fast imaging techniques such as variable flip angle refocusing [50-52]. Although higher field strengths might be able to provide better basic conditions in terms of SNR, the implementation of $3 \mathrm{D}$ acquisition techniques at higher magnetic fields remains challenging. In the field of MS research, there is one study available comparing whole brain 3D FLAIR and T2 TSE sequences showing a better image quality compared to $3 \mathrm{~T}[53]$.

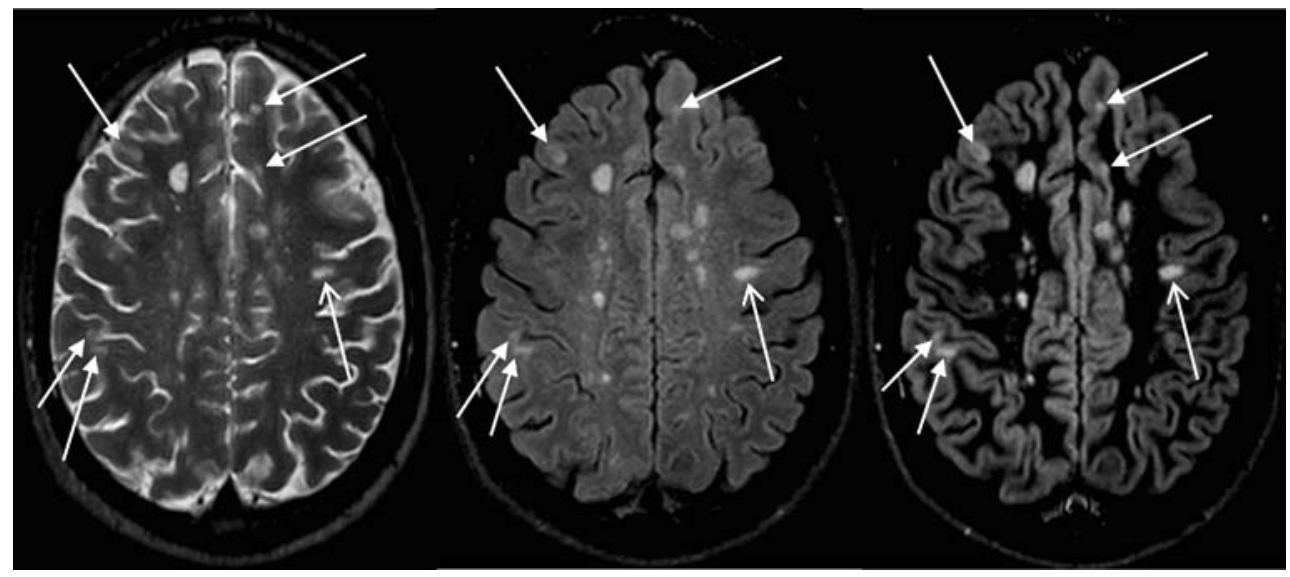

Fig. 5 Transverse sections of a 2D TSE (left), FLAIR (middle), and DIR (right) through the supratentorial brain of a MS patient showing multiple focal lesions in the deep white matter, juxtacortical white matter, and also lesion located partially or totally in the gray matter.
Please note that based on DIR imaging, it is much easier to differentiate between those lesions located in the juxtacortical white matter (open arrow) and those lesions which are at least partially located in the gray matter (closed arrows) 


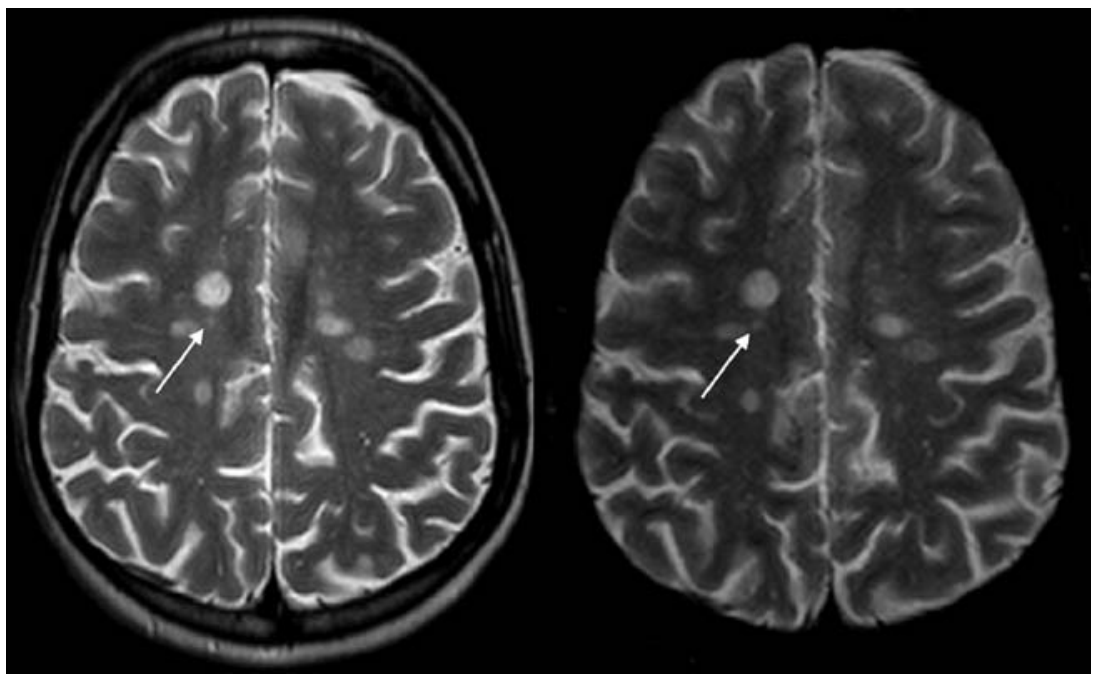

Fig. 6 Axial conventional T2-weighted images within the supratentorial brain of a 41-year-old female with relapsing-remitting MS: on the left side a conventional T2 TSE (acquisition time 2:02 min), on the right side a T2 TSE sequence with variable flip angle refocusing and half-Fourier acquisition (acquisition time 0:11 min). Even small focal lesions could be detected on the speed-optimized T2 TSE sequence (arrow). For detailed sequence parameters, see Lutterbey et al. [49]

\section{Quantitative MRI methods}

The lack of correlation between conventional MRI findings in terms of T2 lesion load and clinical outcome measures, the so-called clinico-radiological dissociation has urged the development of several quantitative MRI methods including MR spectroscopy, diffusion tensor imaging, and magnetization transfer imaging [54-56]. Of special interest are these methods in quantifying disease burden in the normal-appearing brain tissue, particularly in CIS patients in order to get additional prognostic information $[54,57,58]$.

Among those quantitative methods, ${ }^{1} \mathrm{H}-\mathrm{MR}$ spectroscopy benefits the most from the advantages provided by the higher magnetic field strengths $[18,19,59,60]$. Consequently, the most experience concerning quantitative MRI methods in MS patients has been collected for ${ }^{1} \mathrm{H}-\mathrm{MR}$ spectroscopy so far.

\section{${ }^{1} \mathrm{H}-\mathrm{MR}$ spectroscopy}

${ }^{1} \mathrm{H}-\mathrm{MR}$ spectroscopy $\left({ }^{1} \mathrm{H}-\mathrm{MRS}\right)$ is one of the best established quantitative MRI methods allowing the assessment and quantification of metabolic changes in lesions and the normal-appearing brain tissue (normal-appearing white matter; normal-appearing gray matter) of patients with CIS or definite MS [54]. In MS, two major metabolites are of special interest: the first one, the $N$-acetyl moiety $(t N A A)$, a maker of neuronal viability. The second metabolite, myo-inositol ( $m I n s)$, is mostly located in astroglial cells and serves as a marker of glial cell activity [61-64].
In demyelinating diseases, particularly in patients with definite MS, decreased $t N A A$ as well as increased mIns concentrations and therefore axonal damage and increased glial cell activity can be observed in lesions as well as in the normal-appearing brain tissue [65-69]. In patients presenting with CIS, however, significant axonal damage could only be observed in whole brain analysis including normal-appearing brain tissues and lesions but not in the NAWM alone [70]. In contrast, mIns concentrations seem to be increased in the NAWM of CIS patients [71, 72]. All these studies dealing with metabolic alterations in MS and CIS patients were performed at field strengths up to $1.5 \mathrm{~T}$.

${ }^{1} \mathrm{H}-\mathrm{MRS}$ at higher field strengths is characterized by a better spectral separation due to a significant increase in chemical shift particularly for the C4-C6 multiplet of mIns, which at $1.5 \mathrm{~T}$ partly overlaps with the glutamate and glutamine $(G l x)$ signals. Therefore, the combination of an improved spectral separation with an increase in SNR provides a more accurate and reproducible (absolute) quantification of metabolites [18, 73]. Figure 7 gives an example of the altered appearance of the spectrum.

Recently published single voxel ${ }^{1} \mathrm{H}-\mathrm{MRS}$ studies in CIS patients including an optimized acquisition protocol for $t N A A$ and mIns (short and long echo time acquisition) demonstrated that axonal damage is already present in CIS patients not fulfilling the criteria for definite MS [74]. A significant increase of mIns could be observed in patients with an early disease course of relapsing-remitting MS but not in patients presenting with a CIS. However, those CIS patients who converted to definite MS after short-term 


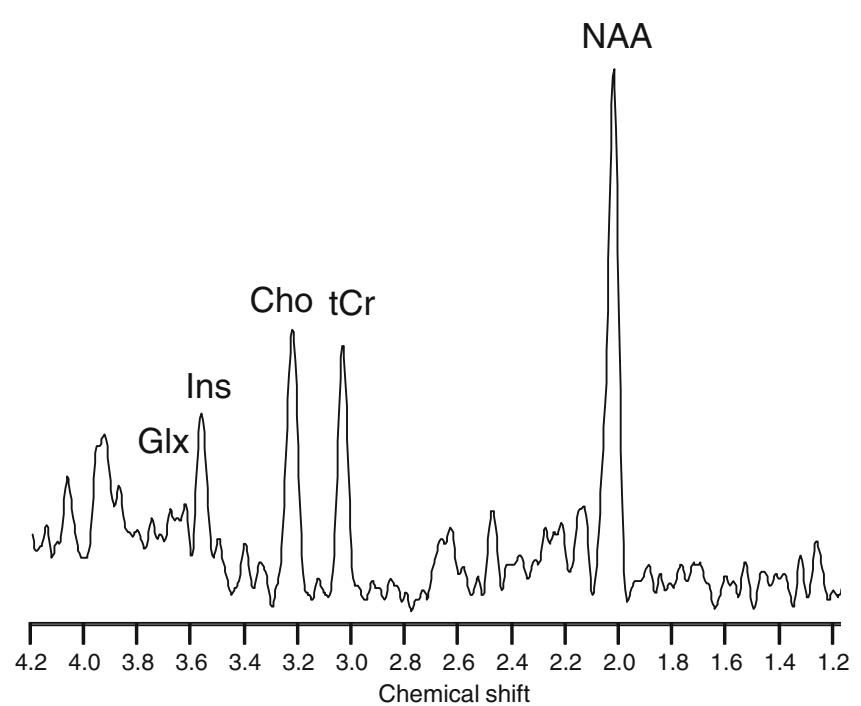

Fig. $7{ }^{1} \mathrm{H}$-MRS spectrum at $1.5 \mathrm{~T}$ (left) and $3 \mathrm{~T}$ (right) obtained from the NAWM of a patient presenting with a CIS suggestive of MS. Please note the change in the appearance at $3 \mathrm{~T}$, especially the signal

follow-up showed almost significantly higher absolute concentrations of $m I n s$ when compared to healthy controls whereas the non-converters did not $(20.2 \%$ versus $1.9 \%$ increase of $m I n s)$. Axonal damage also seems to have prognostic relevance in terms of predicting the conversion to definite MS in CIS patients. MS converters showed evidence of axonal damage in terms of $t N A A$ decrease compared to healthy controls, while non-converters did not [75]. Regarding prognostic purposes, these results obtained at $3 \mathrm{~T}$ are in line with $1.5-\mathrm{T}$ results demonstrating significantly lower $I N A A$ concentration in patients converting to definite MS within 1 year and higher mIns concentration in converters within 3 years time [76].

The "clinico-radiological dissociation" in terms of the non-correlation of lesion load measurements on conventional MRI and clinical outcome measures on one hand and quantitative MRI measures on the other hand can also be observed on magnetic field strengths beyond $1.5 \mathrm{~T}$. Metabolic changes measured with ${ }^{1} \mathrm{H}$-MRS at $3 \mathrm{~T}$ did not correlate with lesion load on conventional MRI finding in terms of fulfilled MRI criteria. Metabolic alterations in the NAWM of CIS patients measured by high field ${ }^{1} \mathrm{H}-\mathrm{MRS}$ which do have a predictive value concerning the conversion to definite MS do not correlate with the fulfilled diagnostic imaging criteria measured by conventional MRI. Even the classification according to the clinical IP criteria in terms of lesion dissemination in space does not correlate with metabolic changes in the NAWM of CIS patients [77]. This once more stresses the fact that changes in the normalappearing brain tissue and lesion load visible on conven-

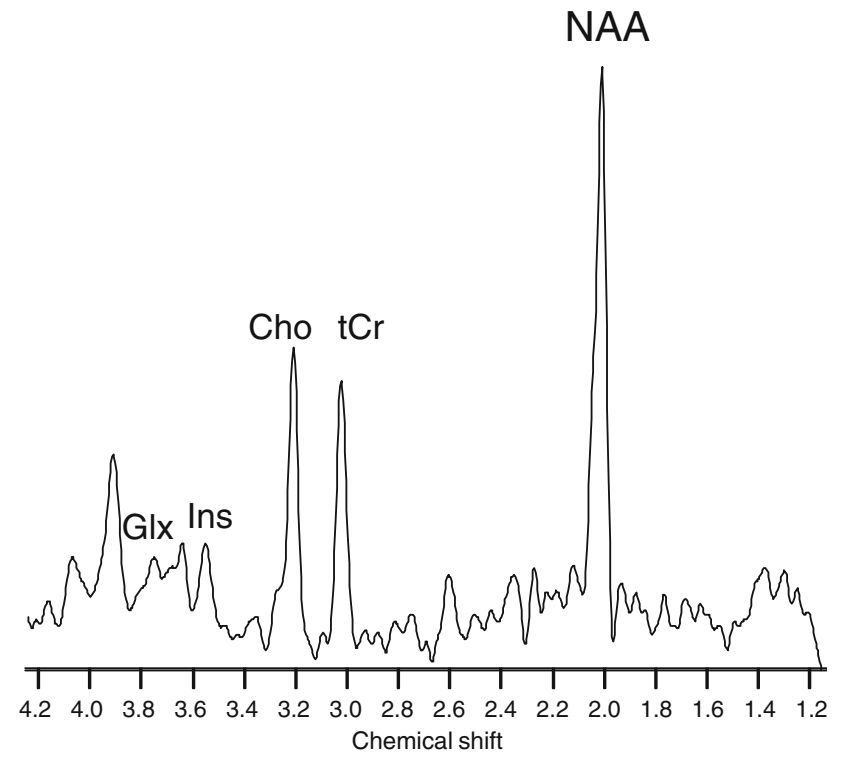

increase and the better separation from $G l x$ for the C4-C6 multiplet of $m I n s$ at $3.63 \mathrm{ppm}$. NAA $\mathrm{N}$-acetylaspartate, $\mathrm{tCr}$ total creatine, Cho choline, Ins (myo)inositol, Glx summation of glutamate and glutamine

tional MRI are developing in different ways and contribute diversely to the natural history of the disease. We have to further investigate cautiously whether advanced MRI methods at higher field strengths such as ${ }^{1} \mathrm{H}-\mathrm{MRS}$ may provide better information concerning the risk of conversion from CIS to definite MS and should be used exclusively or in combination with conventional MRI.

Besides the established metabolites including $t N A A$, $m I n s$, choline $(\mathrm{Cho})$, and total creatine $(\mathrm{tCr})$, further metabolites such as the constituents of Glx signal are gaining certain interest and are beginning to fill voids in the current understanding of pathophysiological processes in MS. Standard field strengths have major difficulties in the (absolute) quantification of Glx. Due to the higher SNR and improved spectral separation at higher fields, an accurate and reproducible quantification of $G l x$ becomes realistic. A recent study applying a TE-averaged PRESS technique at $3 \mathrm{~T}$ showed significantly increased Glutamate concentration in active lesions and also in the NAWM, suggesting that neuro-toxicity expressed by higher concentration of glutamate might play a substantial role in the inducement of axonal damage and therefore clinical disability [78, 79].

Moreover, ${ }^{1} \mathrm{H}$-MRS at $3 \mathrm{~T}$ also offers chances to detect and quantify metabolic changes of CNS regions very important for diagnostic and prognostic purposes, which, however, are difficult to evaluate at lower field strengths because of lower SNR values and the need of high spatial resolution imaging such as the infratentorial brain and the spinal cord [80]. 
Diffusion tensor imaging

Diffusion-weighted imaging in general and diffusion tensor imaging (DTI) in particular have been increasingly applied in the evaluation of pathological changes in MS. Similar to ${ }^{1} \mathrm{H}-\mathrm{MRS}$, DTI is a promising quantitative MRI method for the detection of abnormalities in the normal-appearing brain tissue. It has been consistently shown that altered diffusion in terms of a decreasing fractional anisotropy and increasing mean diffusivity can be observed in lesions and also in the normal-appearing brain tissue $[55,57,81]$.

From the technical point of view, DTI remains a quite challenging method concerning the image acquisition and reproducibility especially across different sequences and scanners (different vendors and magnetic field strengths) [82]. Most DTI sequences are based on single shot echo planar imaging sequences in order to achieve a fast image acquisition. However, this type of sequence is characterized by substantial drawbacks such as a proneness to geometric distortions, limited SNR, and low spatial resolution. High field MRI applications can overcome many of these problems due to the increase of SNR. These higher SNR values can be traded off in a different way: First of all, it allows an image acquisition in a faster way or with a higher spatial resolution (or a combination of both). Secondly, the higher SNR offers the chance to apply parallel imaging techniques which once again can reduce the scan time (due to shorter echo trains), but more importantly also reduces geometrical distortions which consequently lead to a better image quality [83-85]. The higher SNR can also be invested into higher spatial resolution which offers new possibilities for "fiber tracking" applications.

Despite the obvious advantages of DTI at $3 \mathrm{~T}$ in comparison to lower field strengths, the number of published applications in MS is rather limited so far [86-89]. The results of studies performed at $3 \mathrm{~T}$ are not likely to differ substantially from those obtained at lower field strengths, 3 T DTI might offer a greater perspective of (multiparametric) MRI research involving certain anatomic regions which are more difficult to investigate at lower fields such as gray matter structures [86, 87].

\section{Functional MRI}

Susceptibility-weighted imaging (SWI) and BOLD functional MRI (fMRI) are applications which benefit the most from the increased sensitivity to susceptibility differences provided by higher magnetic fields. The BOLD signal increases significantly with the magnetic field strength and therefore allows a better depiction of certain areas of activation which cannot be visualized at standard field strengths.
In MS, fMRI studies of multiple cognitive and motor networks have already shown that abnormal activation and the presence of adaptive properties, the so-called phenomenon of brain plasticity, are already present in the early stages of MS. There have been multiple approaches reported to describe activation patterns and connectivity networks in MS patients across all different stages and disease courses [4, 90, 91]. For instance, for performing simple motor tasks with the right hand, it could be shown that there is an additional activation of the contralateral primary sensorimotor cortex and supplementary motor area. Although the advantage of fMRI based on BOLD contrast at higher fields is quite obvious, the experiences are based on a rather limited number of patients. Using fMRI methods at higher magnetic field strengths, it might be possible to detect new activation patterns and connectivity networks such as those involving the cerebellum and mirror neuron system which cannot easily be evaluated at standard field strengths [92, 93].

\section{Ultra-high field MRI}

High field MRI applications beyond $4 \mathrm{~T}$ are currently considered as ultra-high field MRI. Driven by the success of whole body high field MRI applications at $3 \mathrm{~T}$ and the update of the FDA approval concerning field strengths up to $8 \mathrm{~T}$, we are currently witnessing a rapid increase of installations of ultra-high field MR systems worldwide. Most of them are whole body scanners operating at $7 \mathrm{~T}$ which can be used for post-mortem as well as in vivo applications. However, despite the convincing possibilities in terms of increased SNR, there are substantial challenges such as SAR, inhomogeneities of the magnetic field and the effect on tissue relaxation times. Most of the problems have not been solved so far and remain challenges in particular for in vivo imaging applications.

In MS, ultra-high field MRI was initially used to further visualize and characterize certain aspects of MS pathology at submillimeter resolutions and correlate these findings with the corresponding histopathological results in post-mortem specimen of the brain and the spinal cord. Later on, with the advent of clinical whole-body ultra-high field MRI systems, in vivo studies tried to establish imaging protocols in order to investigate the feasibility of in vivo ultra-high field MR imaging in MS patients despite the technical challenges.

\section{Spinal cord}

Lesions in the spinal cord are frequently observed in MS patients and are already present in early stages of the disease [7, 94, 95]. In contrast to experiences in brain imaging, high field MRI applications up to $3 \mathrm{~T}$ failed to 
demonstrate a higher sensitivity in the detection of spinal cord abnormalities. High resolution post-mortem MR imaging studies at $4.7 \mathrm{~T}$ convincingly showed a high sensitivity in the detection of focal and rather diffuse abnormalities of the spinal cord superior to conventional MRI at 1.0 T with a high grade of correlation regarding the corresponding histopathological results. Furthermore, quantitative MRI methods at $4.7 \mathrm{~T}$ such as the measurement of $\mathrm{T} 1$ relaxation times and $\mathrm{MT}$ ratios showed a high correlation with demyelinating features on the semiquantitative histopathological myelin analysis [96-99].

\section{Brain}

Several high resolution post-mortem MRI studies have already demonstrated the good correlations between imaging findings and histopathological results. Nowadays, there is definitely a need to focus on special pathological features of the disease using ultra-high field MRI. One of the most intriguing imaging and pathological feature of the disease is the detection of cortical abnormalities. Given the fact that conventional MRI in vivo lacks a sufficient sensitivity in visualizing cortical changes, it would be interesting to know whether higher field strengths can overcome this problem. However, the first results using 4.7 $\mathrm{T}$ showed that sensitivity in the detection for cortical lesions remained poor when compared to $1.5 \mathrm{~T}$. Moreover, it has been shown that sensitivity is dependent on the special type of cortical lesions [100]. Moving to field strengths up to $8 \mathrm{~T}$, there is preliminary evidence that the sensitivity might sufficiently increase [101]. However, these findings are based on a very small number of specimens and need to be further validated.
The number of in vivo studies using ultra-high field MRI in MS is very limited so far. Currently, there are no published studies available demonstrating the advantages of ultra-high field MRI in comparison to 1.5 or $3 \mathrm{~T}$. The first in vivo applications of $7 \mathrm{~T}$ MRI in MS were mainly focused on a high resolution imaging of special features of MS pathology such as the perivascular distribution and microvasculature of MS lesions (Fig. 8), a phenomenon which already had been demonstrated at lower field strengths [102, 103].

An intriguing SWI application of ultra-high field MRI at $7 \mathrm{~T}$ which goes beyond the visualization of the microvasculature structures of MS pathology is the implementation of a quantitative MRI method of the local field shift which is based on magnetic susceptibility-shifted compounds of paramagnetic structures such as iron [104, 105]. Since iron plays an important role in acute and chronic inflammatory processes, this technique clearly demonstrated the increased iron deposition in certain structures of MS patients such as the basal ganglia. Moreover, it improves the detection rate of lesions and enables us to visualize in vivo special pathological features such as the iron-rich rim of MS lesions containing iron containing macrophages without injection of any contrast media.

\section{Summary and future directions}

As illustrated in this review, during the past century, overarching research activities have been performed in the area of high field MRI, generally but also specifically in the field of MS research. The question provocatively asked in the title of this article whether higher field leads to higher

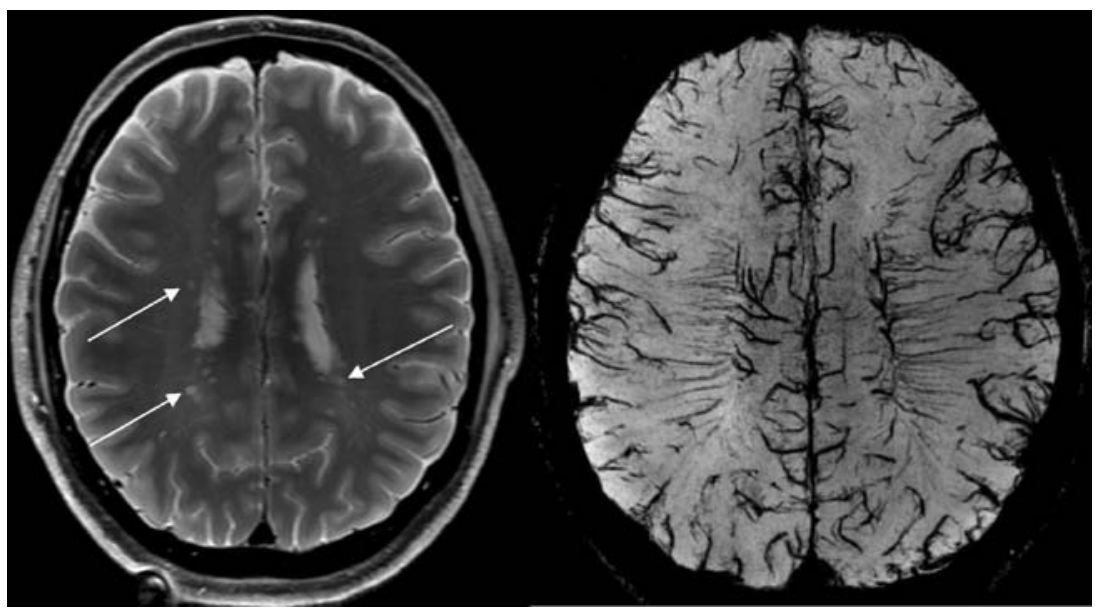

Fig. 8 Axial high resolution T2-weighted image (measured voxel size $0.7 \times 1 \times 2 \mathrm{~mm}$; repetition time $4,969 \mathrm{~ms}$, echo time $80 \mathrm{~ms}$ ) at $7 \mathrm{~T}$ obtained from a patient with relapsing-remitting MS (left side) demonstrating the perivascular distribution of focal MS lesions (arrows). The perivascular distribution pattern can be confirmed by the corresponding (modified) susceptibility-weighted image (measured voxel size $0.35 \times 0.45 \times 0.60 \mathrm{~mm})$ on the right side 
yields can be partially answered with yes. The current generation of high field MRI scanners provides higher sensitivities in the detection of focal abnormalities in the brain, allows the implementation of new pulse sequences such as DIR with improved SNR and positive effect on lesion conspicuity and detectability, delivers substantial improvements for ${ }^{1} \mathrm{H}-\mathrm{MRS}$ in terms of an more accurate and reproducible metabolite quantification, and offers new possibilities for fMRI. However, some important issues have still to be addressed.

One challenging task will be to reveal the potential of high field MR imaging to detect spinal cord abnormalities in MS. Although post-mortem high resolution imaging studies are suggesting the possibility of an increased detection of spinal cord abnormalities, in vivo MRI of the spinal cord remains sophisticated due to many artifacts including CSF and vessel pulsation. In combination with technical challenges, this might be the reason that there are almost no data available so far dealing with the applicability of in vivo high field MRI in the spinal cord of MS patients. Additionally, it is doubtful whether in vivo high field MRI might actually lead to an increased sensitivity in the detection of spinal cord abnormalities. On the other hand, the higher SNR might lead to re-implementation of sequences such as inversion recovery with a low sensitivity at standard field strengths.

Most of the research has focused on conventional brain imaging in MS, and across all studies, it has been conclusively shown that brain imaging benefits most from the higher field strengths. However, there are still important questions to address. One of these deals with the visualization of cortical abnormalities. It is important to know whether 3-T MRI in vivo using DIR is able to detect more cortical abnormalities than $1.5 \mathrm{~T}$ does. In a second step, we have to try to implement DIR sequences also into in vivo ultra-high field (7 T) MRI protocols and attempt to further increase the sensitivity regarding the detection of cortical abnormalities because these changes are a hallmark of MS pathology. Additionally, higher magnetic fields might lead to a better detection and classification of diffuse abnormalities in MS, a radiological feature which is probably underrated in many ways so far.

New more specific contrast media are coming up in the in vivo detection of active MS pathology and are currently evaluated in terms of standard magnetic field strengths. Especially substances based on iron oxide such as ultra-small particles of iron oxide indicating a cellular inflammation mediated by macrophages might benefit substantially from the higher magnetic fields using special imaging techniques such as susceptibility-weighted imaging.

Acknowledgments The authors would like to thank Dr. Frank Träber for providing Fig. 7, Jürgen Gieseke for providing Fig. 6,
Wolter de Graaf for providing Fig. 8, and Dr. Alexandra Seewann for reviewing this manuscript.

Conflict of interest statement We declare that we have no conflict of interest.

Open Access This article is distributed under the terms of the Creative Commons Attribution Noncommercial License which permits any noncommercial use, distribution, and reproduction in any medium, provided the original author(s) and source are credited.

\section{References}

1. Pugliatti M, Rosati G, Carton H et al (2006) The epidemiology of multiple sclerosis in Europe. Eur J Neurol 13:700-722. doi:10.1111/j.1468-1331.2006.01342.x

2. Noseworthy JH, Lucchinetti C, Rodriquez M, Weinshenker BG (2000) Multiple sclerosis. N Engl J Med 28:938-952. doi:10.1056/NEJM200009283431307

3. Miller DH, Filippi M, Fazekas F, Frederiksen JL, Matthews PM, Montalban X, Polman CH (2004) Role of magnetic resonance imaging within diagnostic criteria for multiple sclerosis. Ann Neurol 56:272-278

4. Bakshi R, Thompson AJ, Rocca MA et al (2008) MRI in multiple sclerosis: current status and future prospects. Lancet Neurol 7:615-625. doi:10.1016/S1474-4422(08)70137-6

5. Rovira Cañellas A, Rovira Gols A, Rio Izquierdo J, Tintoré Subirana M, Montalban Gairin X (2007) Idiopathic inflammatorydemyelinating diseases of the central nervous system. Neuroradiology 49:393-409. doi:10.1007/s00234-007-0216-2

6. McDonald WI, Compston A, Edan G et al (2001) Recommended diagnostic criteria for multiple sclerosis: guidelines from the International Panel on the diagnosis of multiple sclerosis. Ann Neurol 50:121-127. doi:10.1002/ana.1032

7. Polman CH, Reingold SC, Edan G et al (2005) Diagnostic Criteria for Multiple Sclerosis: 2005 Revisions to the "McDonald Criteria". Ann Neurol 58:840-846. doi:10.1002/ana.20703

8. Barkhof F, Filippi M, Miller DH et al (1997) Comparison of MRI criteria at first presentation to predict conversion to clinically definite multiple sclerosis. Brain 120:2059-2069. doi:10.1093/ brain/120.11.2059

9. Tintoré M, Rovira A, Martinez MJ et al (2000) Isolated demyelinating syndromes: comparison of different MR imaging criteria to predict conversion to clinically definite multiple sclerosis. AJNR Am J Neuroradiol 21:702-706

10. Minneboo A, Barkhof F, Polman CH et al (2004) Infratentorial lesions predict long-term disability in patients with initial findings suggestive of multiple sclerosis. Arch Neurol 61:217221. doi:10.1001/archneur.61.2.217

11. U.S. Department of Health and Human Services, Food and Drug Administration, Center for Devices and Radiological Health, Radiological Devices Branch, Division of Reproductive, Abdominal, and Radiological Devices, Office of Device Evaluation. Guidance for industry and FDA staff: criteria for significant risk investigations of magnetic resonance diagnostic devices. http:// www.fda.gov/cdrh/ode/guidance/793.html. Updated July 14, 2003. Accessed 18 Sept 2008

12. Machann J, Schlemmer HP, Schick F (2008) Technical challenges and opportunities of whole body magnetic resonance imaging at 3 T. Phys Med 18:1449-1455

13. Schick F (2005) Whole-body MRI at high field: technical limits and clinical potential. Eur Radiol 15:946-959. doi:10.1007/ s00330-005-2678-0 
14. Willinek WA, Kuhl CK (2006) 3.0 T neuroimaging: technical considerations and clinical applications. Neuroimaging Clin $\mathrm{N}$ Am 16:217-228. doi:10.1016/j.nic.2006.02.007

15. Willinek WA, Schild HH (2007) Clinical advantages of $3.0 \mathrm{~T}$ MRI over 1.5 T. Eur J Radiol 65:2-14. doi:10.1016/j.ejrad. 2007.11.006

16. Krautmacher C, Willinek WA, Tschampa HJ et al (2005) Brain tumors: full- and half-dose-contrast-enhanced MR imaging at 3.0 T compared with 1.5 T-initial experience. Radiology 237:1014-1019. doi:10.1148/radiol.2373041672

17. Wansapura JP, Holland SK, Dunn RS et al (1999) NMR relaxation times in the human brain at 3.0 T. J Magn Reson Imaging 9:531-538. doi:10.1002/(SICI) 1522-2586(199904)9:4 $<531::$ AID-JMRI4>3.0.CO;2-L

18. Di Constanzo A, Trosji F, Tosetti M et al (2007) Proton MR spectroscopy of the brain. An update. Eur Radiol 17:1651-1662. doi:10.1007/s00330-006-0546-1

19. Jansen JFA, Backes WH, Nicolay K, Kooi ME (2006) 1H MR spectroscopy of the brain: absolute quantification of metabolites. Radiology 240:318-332. doi:10.1148/radiol.2402050314

20. U.S. Department of health and human services, Food and Drug Administration, Center for Devices and Radiological Health, A Primer on Medical Device Interactions with Magnetic Resonance Imaging Systems. http://www.fda.gov/cdrh/ode/primerf6.html. Assessed 18 Sept 2008

21. Schima W, Wimberger D, Schneider B, Stiglbauer R, Asenbaum S, Imhof H (1993) The importance of magnetic field strength in the MR diagnosis of multiple sclerosis: a comparison of $0.5 \mathrm{~T}$ and 1.5 T. Rofo 158:368-371. doi:10.1055/s-2008-1032665

22. Lee DH, Vellet AD, Eliasziw M et al (1995) MR imaging field: prospective evaluation of the diagnostic accuracy of MR for diagnosis of multiple sclerosis at 0.5 and $1.5 \mathrm{~T}$. Radiology 194:257-262

23. Filippi M, van Waesberghe JH, Horsfield MA et al (1997) Interscanner variation in brain MRI lesion load measurements in MS. Neurology 49:371-377

24. Poser CM, Paty DW, Scheinberg L et al (1983) New diagnostic criteria for multiple sclerosis: guidelines for research protocols. Ann Neurol 13:227-231. doi:10.1002/ana.410130302

25. Keiper MD, Grossmann RI, Hirsch JA et al (1998) MR identification of white matter abnormalities in multiple sclerosis: a comparison between $1.5 \mathrm{~T}$ and $4 \mathrm{~T}$. AJNR Am J Neuroradiol 19:1489-1493

26. Sicotte NL, Voskuhl RR, Bouvier S, Klutch R, Cohen MS, Mazziotta JC (2003) Comparison of multiple sclerosis lesions at 1.5 and 3.0 T. Invest Radiol 38:423-427. doi:10.1097/00004424200307000-00007

27. Wattjes MP, Lutterbey GG, Harzheim M et al (2006) Higher sensitivity in the detection of inflammatory brain lesions in patients with clinically isolated syndromes suggestive of multiple sclerosis using high field MRI: an intraindividual comparison of 1.5 T with 3.0 T. Eur Radiol 16:2067-2073. doi:10.1007/s00330006-0195-4

28. Nielsen K, Rostrup E, Frederiksen JL et al (2006) Magnetic resonance imaging at 3.0 Tesla detects more lesions in acute optic neuritis than at 1.5 Tesla. Invest Radiol 41:76-82. doi:10.1097/01.rli.0000188364.76251.28

29. Bachmann R, Reilmann R, Schwindt W, Kugel H, Heindel W, Krämer S (2006) FLAIR imaging for multiple sclerosis: a comparative MR study at 1.5 and 3.0 Tesla. Eur Radiol 16:915-921. doi:10.1007/s00330-005-0070-8

30. Wattjes MP, Harzheim M, Kuhl CK et al (2006) Does high-field MRI have an influence on the classification of patients with clinically isolated syndromes according to current diagnostic magnetic resonance imaging criteria for multiple sclerosis? AJNR Am J Neuroradiol 27:1794-1798
31. Wattjes MP, Harzheim M, Lutterbey GG et al (2008) Does high field MRI allow an earlier diagnosis of multiple sclerosis? J Neurol 50:1159-1163. doi:10.1007/s00415-008-0861-3

32. Swanton JK, Rovira A, Tintore M et al (2007) MRI criteria for multiple sclerosis in patients presenting with clinically isolated syndromes: a multicentre retrospective study. Lancet Neurol 6:677-686. doi:10.1016/S1474-4422(07)70176-X

33. Stankiewicz JM, Neema M, Alsop DC et al (2009) Spinal cord lesions and clinical status in multiple sclerosis: a $1.5 \mathrm{~T}$ and $3 \mathrm{~T}$ MRI study. J Neurol Sci 279:99-105

34. Simon JH, Li D, Traboulsee A, Arnold DL et al (2006) Standardized MR imaging protocol for multiple sclerosis: consortium of MS centers consensus guidelines. AJNR Am J Neuroradiol 27:455-461

35. Yousry TA, Filippi M, Becker C, Horsfield MA, Voltz R (1997) Comparison of MR pulse sequences in the detection of multiple sclerosis lesions. AJNR Am J Neuroradiol 18:959-963

36. Frohman EM, Zhang H, Kramer PD et al (2001) MRI chararcteristics of MLF in MS patients with chronic internuclear ophthalmoparesis. Neurology 57:762-768

37. Filippi M, Yousry T, Baratti C et al (1996) Quantitative assessment of MRI lesion load in multiple sclerosis: a comparison of conventional spin-echo with fast fluid-attenuated inversion recovery. Brain 119:1349-1355. doi:10.1093/brain/119.4.1349

38. Gawne-Cain ML, O'Riordan JI, Thompson AJ et al (1997) Multiple sclerosis lesion detection in the brain: a comparison of fast fluid-attenuated inversion recovery and conventional T2weighted dual spin echo. Neurology 49:364-370

39. Bakshi R, Ariyaratana S, Benedict RH, Jacobs L (2001) Fluidattenuated inversion recovery magnetic resonance imaging detects cortical and juxtacortical multiple sclerosis lesions. Arch Neurol 58:742-748. doi:10.1001/archneur.58.5.742

40. Wattjes MP, Lutterbey GG, Harzheim M et al (2006) Imaging of inflammatory lesions at 3.0 Tesla in patients with clinically isolated syndromes suggestive of multiple sclerosis: a comparison of fluid-attenuated inversion recovery with T2 turbo spin-echo. Eur Radiol 16:1494-1500. doi:10.1007/s00330-005-0082-4

41. Redpath TW, Smith FW (1994) Technical note: use of a double inversion recovery pulse sequence to image selectively grey or white brain matter. Br J Radiol 67:1258-1263

42. Bedell BJ, Narayana PA (1998) Implementation and evaluation of a new pulse sequence for rapid acquisition of double inversion recovery images for simultaneous suppression of white matter and CSF. J Magn Reson Imaging 8:544-547. doi:10.1002/ jmri. 1880080305

43. Turetschek K, Wunderbaldinger P, Bankier AA, Zontsich T, Graf O, Mallek R, Hittmair K (1998) Double inversion recovery imaging of the brain: initial experience and comparison with fluid attenuated inversion recovery imaging. Magn Reson Imaging 16:127-135. doi:10.1016/S0730-725X(97)00254-3

44. Geurts JJG, Pouwels PJW, Uitdehaag BMJ, Polman CH, Barkhof F, Castelijns JA (2005) Intracortical lesions in multiple sclerosis: improved detection with double inversion-recovery MR imaging. Radiology 236:254-260. doi:10.1148/radiol.2361040450

45. Kidd D, Barkhof F, McConnell R, Algra PR, Allen IV, Revesz T (1999) Cortical lesions in multiple sclerosis. Brain 122:17-26. doi:10.1093/brain/122.1.17

46. Calabrese M, De Stefano N, Atzori M et al (2007) Detection of cortical inflammatory lesions by double inversion recovery magnetic resonance imaging in patients with multiple sclerosis. Arch Neurol 64:1416-1422. doi:10.1001/archneur.64.10.1416

47. Calabrese M, De Stefano N, Atzori M et al (2008) Extensive cortical inflammation is associated with epilepsy in multiple sclerosis. J Neurol 255:581-586. doi:10.1007/s00415-008-0752-7

48. Wattjes MP, Lutterbey GG, Gieseke J et al (2007) Double inversion-recovery brain imaging at 3.0 Tesla: diagnostic value 
in the detection of multiple sclerosis lesions. AJNR Am J Neuroradiol 28:54-59. doi:10.3174/ajnr.A0594

49. Lutterbey G, Wattjes MP, Kandyba J et al (2007) Clinical evaluation of a speed optimized T2 weighted fast spin echo sequence at 3.0 $\mathrm{T}$ using variable flip angle refocusing, halfFourier acquisition and parallel imaging. Br J Radiol 80:668673. doi:10.1259/bjr/88996134

50. Pouwels PJ, Kuijer JP, Mugler JP 3rd, Guttmann CR, Barkhof F (2006) Human gray matter: feasibility of a single-slap 3D double inversion recovery high spatial resolution MR imaging. Radiology 241:873-879. doi:10.1148/radiol.2413051182

51. Mugler JP, Bao S, Mulkern RV et al (2000) Optimized singleslab three-dimensional spin-echo MR imaging of the brain. Radiology 216:891-899

52. Bagnato F, Butman JA, Gupta S et al (2006) In vivo detection of cortical plaques by MR imaging in patients with multiple sclerosis. AJNR Am J Neuroradiol 27:2161-2167

53. Mills RJ, Young CA, Smith ET (2007) 3D MRI in multiple sclerosis: a study of three sequences at $3 \mathrm{~T}$. Br J Radiol 80:307320. doi: $10.1259 / \mathrm{bjr} / 52670770$

54. Miller DH, Thompson AJ, Filippi M (2003) Magnetic resonance studies of abnormalities in the normal appearing white matter and grey matter in multiple sclerosis. J Neurol 250:1407-1419. doi:10.1007/s00415-003-0243-9

55. Pagani E, Bammer R, Horsfield MA et al (2007) Diffusion MR imaging in multiple sclerosis: technical aspects and challenges. AJNR Am J Neuroradiol 28:411-420

56. Ge Y, Grossman RI, Udupa JK et al (2001) Magnetization transfer ratio histogram analysis of gray matter in relapsingremitting multiple sclerosis. AJNR Am J Neuroradiol 22:470475

57. Bester M, Heesen C, Schippling S et al (2008) Early anisotropy changes in the corpus callosum of patients with optic neuritis. Neuroradiology 50:549-557. doi:10.1007/s00234-008-0377-7

58. Fernando KTM, Tozer DJ, Mizkiel KA et al (2005) Magnetization transfer histograms in clinically isolated syndromes suggestive of multiple sclerosis. Brain 128:2911-2925. doi:10.1093/ brain/awh654

59. Träber F, Block W, Lamerichs R, Gieseke J, Schild HH (2004) $1 \mathrm{H}$ metabolite relaxation times at 3.0 Tesla: measurements of T1 and $\mathrm{T} 2$ values in normal brain and determination of regional differences in transverse relaxation. J Magn Reson Imaging 19:537-545. doi:10.1002/jmri.20053

60. Gonen O, Gruber S, Mi BS et al (2001) Multivoxel 3D proton spectroscopy in the brain at 1.5 versus $3.0 \mathrm{~T}$ : signal-to-noise ratio and resolution comparison. AJNR Am J Neuroradiol 22:17271731

61. Bitsch A, Bruhn H, Vougioukas V et al (1999) Inflammatory CNS demyelination: histopathologic correlation with in vivo quantitative proton MR spectroscopy. AJNR Am J Neuroradiol 20:1619-1627

62. Simmons ML, Frondonza CG, Coyle JT (1991) Immunocytochemical localization of $\mathrm{N}$-acetyl-aspartate with monoclonal antibodies. Neuroscience 45:37-45. doi:10.1016/0306-4522(91) 90101-S

63. Rigotti DJ, Inglese $\mathrm{M}$, Gonen $\mathrm{O}$ (2007) Whole-brain $\mathrm{N}$ acetylaspartate as a surrogate marker of neuronal damage in diffuse neurologic disorders. AJNR Am J Neuroradiol 28:18431849. doi:10.3174/ajnr.A0774

64. Brand A, Richter-Landsberg C, Leibfritz D (1993) Multinuclear NMR studies on the energy metabolism of glial and neuronal cells. Dev Neurosci 15:289-298. doi:10.1159/000111347

65. Chard DT, Griffin CM, McLean MA et al (2002) Brain metabolite changes in cortical grey and normal-appearing white matter in clinically early relapsing remitting multiple sclerosis. Brain 125:2342-2352. doi:10.1093/brain/awf240
66. Kapeller P, McLean MA, Griffin CM et al (2001) Preliminary evidence for neuronal damage in cortical grey and normal appearing white matter in short duration relapsing-remitting multiple sclerosis: a quantitative MR spectroscopic imaging study. J Neurol 248:131-138. doi:10.1007/s004150170248

67. Tedeschi G, Bonavita S, McFarland HF, Richert N, Duyn JH, Frank JA (2002) Proton MR spectroscopic imaging in multiple sclerosis. Neuroradiology 44:37-42. doi:10.1007/s002340 100584

68. Tiberio M, Chard DT, Altmann DR et al (2006) Metabolite changes in early relapsing-remitting multiple sclerosis. A two year follow-up study. J Neurol 253:224-230. doi:10.1007/ s00415-005-0964-z

69. Drehe-Kulaczewski SF, Helms G, Dechent P et al (2009) Serial proton MR spectroscopy and diffusion tensor imaging in infantile Balo's concentric sclerosis. Neuroradiology 51:113121. doi:10.1007/s00234-008-0470-y

70. Filippi M, Bozzali M, Rovaris M et al (2003) Evidence for widespread axonal damage at the earliest clincial stage of multiple sclerosis. Brain 126:433-437. doi:10.1093/brain/awg038

71. Tourbah A, Stievenart JL, Abanou A et al (1999) Normalappearing white matter in optic neuritis and multiple sclerosis: a comparative proton spectroscopy study. Neuroradiology 41:738743. doi:10.1007/s002340050835

72. Fernando KTM, McLean MA, Chard DT et al (2004) Elevated white matter myo-inositol in clinical isolated syndromes suggestive of multiple sclerosis. Brain 127:1361-1369. doi:10.1093/ brain/awh153

73. Srinivasan R, Vignerion D, Sailasuta N, Hurd R, Nelson S (2004) A comparative study of myo-inositol quantification using LC model at $1.5 \mathrm{~T}$ and $3.0 \mathrm{~T}$ with $3 \mathrm{D} 1 \mathrm{H}$ proton spectroscopic imaging of the human brain. Magn Reson Imaging 22:523-528. doi:10.1016/j.mri.2004.01.028

74. Wattjes MP, Harzheim M, Lutterbey G, Klotz L, Schild HH, Träber F (2007) Axonal damage but no increased glial cell activity in the normal-appearing white matter of patients with clinically isolated syndromes suggestive of multiple sclerosis using high field magnetic resonance spectroscopy. AJNR Am J Neuroradiol 28:1517-1522. doi:10.3174/ajnr.A0594

75. Wattjes MP, Harzheim M, Lutterbey GG, Bogdanow M, Schmidt S, Schild HH, Träber F (2008) Prognostic value of high field proton magnetic resonance spectroscopy in patients presenting with clinically isolated syndromes suggestive of multiple sclerosis. Neuroradiology 50:123-129. doi:10.1007/ s00234-007-0325-y

76. Fernando KTM, Chard DT, McLean MA (2006) Metabolite changes in the white matter 3 years after a clinically isolated syndrome suggestive of multiple sclerosis. Mult Scler 12 (S1):41

77. Wattjes MP, Harzheim M, Lutterbey GG, Bogdanow M, Schmidt S, Schild HH, Träber F (2008) High field MR imaging and 1HMR spectroscopy in clinically isolated syndromes suggestive of multiple sclerosis: correlation between metabolic alterations and diagnostic MR imaging criteria. J Neurol 255:56-63. doi:10.1007/s00415-007-0666-9

78. Hurd R, Sailasuta N, Srinivasan R et al (2004) Measurement of brain glutamate using T2-averraged PRESS at 3 T. Magn Reson Med 51:435-440. doi:10.1002/mrm.20007

79. Srinivasan R, Sailasuta N, Hurd R, Nelson S, Pelletier D (2005) Evidence of elevated glutamate in multiple sclerosis using magnetic resonance spectroscopy at 3 T. Brain 128:1016-1025. doi:10.1093/brain/awh467

80. Marliani AF, Clementi V, Albini-Riccoli L et al (2007) Quantitative proton magnetic resonance spectroscopy of the human cervical spinal cord at 3 Tesla. Magn Reson Med 57:160 163. doi:10.1002/mrm.21113 
81. Rovaris M, Gass A, Bammer R et al (2005) Diffusion MRI in multiple sclerosis. Neurology 65:1526-1532. doi:10.1212/01. wnl.0000184471.83948.e0

82. Lee CE, Danielian LE, Thomasson D, Baker EH (2009) Normal regional fractional anisotropy and apparent diffusion coefficient of the brain measured on a $3 \mathrm{~T}$ MR scanner. Neuroradiology 51:3-9. doi:10.1007/s00234-008-0441-3

83. Pruessmann KP, Weiger $M$, Scheidegger MB, Boesinger $P$ (1999) SENSE : sensitivity encoding for fast MRI. Magn Reson Med 42:952-962. doi:10.1002/(SICI) 1522-2594(199911) 42:5<952::AID-MRM16>3.0.CO;2-S

84. Jaermann T, Crelier G, Pruessmann KP et al (2004) SENSE-DTI at 3 T. Magn Reson Med 51:230-236. doi:10.1002/mrm.10707

85. Kuhl CK, Gieseke J, von Falkenhausen M et al (2005) Sensitivity encoding for diffusion-weighted MR imaging at 3.0 T: intraindividual comparative study. Radiology 234:517526. doi:10.1148/radiol.2342031626

86. Ceccarelli A, Rocca MA, Pagani E et al (2008) The topographical distribution of tissue injury in benign MS at $3 \mathrm{~T}$ : a multiparametric MRI study. Neuroimage 15(39):1499-1509. doi:10.1016/j.neuro image.2007.11.002

87. Ceccarelli A, Rocca MA, Falini A et al (2007) Normal appearing white and grey matter damage in multiple sclerosis in MS: a volumetric and diffusion tensor MRI study at 3.0 Tesla. J Neurol 254:513-518. doi:10.1007/s00415-006-0408-4

88. Reich DS, Zackowski KM, Gordon-Lipkin EM, Smith SA et al (2008) Corticospinal tract abnormalities are associated with weakness in multiple sclerosis. AJNR Am J Neuroradiol 29:333-339. doi:10.3174/ajnr.A0788

89. Naismith RT, Xu J, Tutlam NT et al (2009) Disability in optic neuritis correlates with diffusion tensor-derived directional diffusivities. Neurology 72:589-594

90. Rovira A, León A (2008) MR in the diagnosis and monitoring of multiple sclerosis: an overview. Eur J Radiol 67:409-414. doi:10.1016/j.ejrad.2008.02.044

91. Rocca MA, Filippi M (2007) Functional MRI in multiple sclerosis. J Neuroimaging 1:36-41

92. Rocca MA, Pagani E, Absinta M et al (2007) Altered functional and structural connectivities in patients with MS: a 3-T study. Neurology 69:2136-2145. doi:10.1212/01.wnl.0000295504.92020.ca

93. Rocca MA, Tortorella P, Ceccarelli A et al (2008) The "mirrorneuron-system" in MS. A 3 Tesla fMRI study. Neurology 70:255-262. doi:10.1212/01.wnl.0000284667.29375.7e

94. Bot JC, Barkhof F, Lycklama à Nijeholt G et al (2002) Differentiation of multiple sclerosis from other inflammatory disorders and cerebrovascular disease: value of spinal MR imaging. Radiology 223:46-56. doi:10.1148/radiol.2231010707

95. Dalton CM, Brex PA, Miszkiel KA et al (2003) Spinal cord MRI in clinically isolated optic neuritis. J Neurol Neurosurg Psychiatry 74:1577-1580. doi:10.1136/jnnp. 74.11.1577

96. Lycklama à Nijeholt GJ, Bergers E, Kamphorst W et al (2001)) Post-mortem high-resolution MRI of the spinal cord in multiple sclerosis: a correlative study with conventional MRI, histopathology and clinical phenotype. Brain 124:154-166

97. Bot JCJ, Blezer ELA, Kamphorst W et al (2004) The spinal cord in multiple sclerosis: relationship of high-spatial-resolution quantitative MR imaging findings to histopathologic results. Radiology 233:531-540. doi:10.1148/radiol.2332031572

98. Mottershead JP, Schmierer K, Clemence M et al (2003) High field MRI correlates of myelin content and axonal density in multiple sclerosis - a post-mortem study of the spinal cord. J Neurol 250:1293-1301. doi:10.1007/s00415-003-0192-3

99. Gilmore CP, Geurts JJG, Evangelou N et al (2009) Spinal cord grey matter lesions in multiple sclerosis detected by post-mortem high field MR imaging. Mult Scler 15:180-188. doi:10.1177/ 1352458508096876

100. Geurts JJG, Blezer EL, Vrenken H et al (2008) Does high-field MR imaging improve cortical lesion detection in multiple sclerosis. J Neurol 255:183-191. doi:10.1007/s00415-0080620-5

101. Kangarlu A, Bourekas EC, Ray-Chaudhury A, Rammohan KW (2007) Cerebral cortical lesions in multiple sclerosis detected by MR imaging at 8 Tesla. AJNR Am J Neuroradiol 28:262-266

102. Ge Y, Zohrabian VM, Grossman RI (2008) Seven-Tesla magnetic resonance imaging: new vision of microvascular abnormalities in multiple sclerosis. Arch Neurol 65:812-816. doi:10.1001/archneur.65.6.812

103. Tallentyre EC, Brookes MJ, Dixon JE et al (2008) Demonstrating the perivascular distribution of MS lesions in vivo with 7-Tesla MRI. Neurology 70:2076-2078. doi:10.1212/01.wnl.000031 3377.49555.2e

104. Hammond KE, Lupo JM, Xu D et al (2008) Development of a robust method for generating 7.0 T multichannel phase images of the brain with application to normal volunteers and patients with neurological diseases. Neuroimage 39:1682-1692. doi:10.1016/j. neuroimage.2007.10.037

105. Hammond KE, Metcalf M, Carvajal L et al (2008) Quantitative in vivo magnetic resonance imaging of multiple sclerosis at 7 Tesla with sensitivity to iron. Ann Neurol 64:707-713. doi:10.1002/ana.21582 